\title{
1 Adaptation and competition in deteriorating environments
}

2

3 Romana Limberger ${ }^{1,2,3^{*}}$ and Gregor F. Fussmann ${ }^{2}$

4

51 Research Department for Limnology, University of Innsbruck, Mondsee, Austria

62 Department of Biology, McGill University, Montreal, Quebec, Canada

73 Department of Evolutionary Biology and Environmental Studies, University of Zurich,

$8 \quad$ Zurich, Switzerland

9

10 E-mail addresses:

11 romana.limberger@ieu.uzh.ch

12 gregor.fussmann@mcgill.ca

13

$14 *$ Corresponding author:

15 Romana Limberger

16 Department of Evolutionary Biology and Environmental Studies

17 Winterthurerstrasse 190, 8057 Zurich, Switzerland

18

e-mail:romana.limberger@ieu.uzh.ch

19

20 Key words: abiotic stressor, adaptation, competition, eco-evolutionary dynamics,

21 environmental change, experimental evolution 


\section{Abstract}

25 Evolution might rescue populations from extinction in changing environments. Using experimental evolution with microalgae, we investigated if competition influences adaptation to an abiotic stressor, and vice versa, if adaptation to abiotic change influences competition. In a first set of experiments, we propagated monocultures of five species with and without increasing salt stress for $\sim 180$ generations. When assayed in monoculture, two of the five species showed signatures of adaptation, that is, lines with a history of salt stress had higher population growth rates at high salt than lines without prior exposure to salt. When assayed in mixtures of species, however, only one of these two species had increased population size at high salt, indicating that competition can alter how adaptation to abiotic change influences population dynamics. In a second experiment, we cultivated two species in monocultures and in pairs, with and without increasing salt. While we found no effect of competition on adaptation to salt, our experiment revealed that evolutionary responses to salt can influence competition. Specifically, one of the two species had reduced competitive ability in the no-salt environment after long-term exposure to salt stress. Collectively, our results highlight the complex interplay of adaptation to abiotic change and competitive interactions. 


\section{Introduction}

Abiotic environmental change can render populations maladapted, leading to their decline, and potentially, extinction [1-3]. Population decline may be slowed or arrested by adaptation $[4,5]$, that is, by phenotypic change that improves fitness and has a genetic basis [6]. Both laboratory and field-based studies have demonstrated that organisms can adapt rapidly to a variety of abiotic drivers, including acidification [7], salt stress [8], pollution [9], nutrient limitation [10], and warming [11, 12]. Experiments have also demonstrated evolutionary responses to biotic drivers, such as competition [13-15] and predation [16]. However, only few experiments have explored the interactive effects of abiotic change and biotic interactions on adaptation [16-19]. Yet, species interactions might influence (i) whether populations adapt to abiotic change [16-18], and (ii) whether adaptation increases population size and persistence [20]. Moreover, within communities, the ecological consequences of adaptation to abiotic change could go beyond effects on population size, and include effects on species interactions and community dynamics [21]. This potentially complex interplay of biotic interactions and adaptation to abiotic change limits our understanding of whether evolution can contribute to the maintenance of diversity in changing environments.

Theory suggests several mechanisms whereby interspecific competition can influence adaptation to deteriorating environmental conditions. First, competition can constrain adaptation to environmental change by reducing population size, and thus the amount of standing genetic variation [22] and the supply of beneficial mutations [23]. Second, competition can reduce the time available for adaptation as species are outcompeted by competitors with broader environmental tolerance [24]. Third, competition can alter the strength and direction of selection [25]; depending on whether competition and environmental change impose selection on a trait in the same or in the opposite direction, competition can speed up or hinder adaptation to environmental change [25]. Finally, negative genetic 
correlations between traits that mediate competition and stress tolerance could constrain adaptation in environments with both biotic and abiotic selective agents [26]. Only few experiments have analysed evolutionary responses to a factorial manipulation of abiotic change and biotic interactions to test these theoretical predictions [16-19]. Moreover, most of these empirical tests investigated biotic interactions other than interspecific competition [16, 17], or environmental amelioration rather than deterioration [17, 18, but see 19]. In our study, however, we focused on interspecific competition, a key biotic interaction, and its interplay with adaptation to deteriorating conditions, that is, to environmental changes that have a negative effect on fitness (e.g. acidification, pollution).

Competition could not only alter how species adapt to abiotic change, but could also modulate the effect of adaptation on population dynamics. When competition alters the fitness landscape generated by abiotic change, adaptation of a focal species in isolation may not translate into increased population size in a community context. Accordingly, an experiment that manipulated $\mathrm{CO}_{2}$ and diversity of a plant community found adaptation to elevated $\mathrm{CO}_{2}$ only when plants were assayed in the same community context in which selection had occurred [18]. Similarly, an experiment with a marine alga revealed that adaptation to $\mathrm{CO}_{2}$ enrichment in isolation was not associated with increased population size within communities [20]. The scenario of adaptation in isolation followed by competition might seem artificial, but it is a plausible scenario in a spatial context. Species could adapt to changes in their local environment before immigration of better-adapted competitors from the regional species pool $[27,28]$. Such changes in competitive interactions could prevent that adaptation of a focal species to abiotic change translates into increased population size.

When investigating the ecological consequences of adaptation to an abiotic stressor, the focus is often on effects on population size and persistence, but within communities, adaptation of a focal species to the local environment may affect interactions with other 
species [29-31]. Evolution can influence community dynamics directly, by altering traits that underlie species interactions, and indirectly, by influencing population dynamics $[32,33]$. In a scenario with an abiotic stressor as the selective agent, adaptation could influence species interactions if traits that confer adaptation to the stressor are correlated with traits that affect biotic interactions. Some traits may even simultaneously mediate stress tolerance and species interactions. For example, in E. coli reduced membrane permeability increases resistance to antibiotics but decreases the ability to take up nutrients, such that adaptation to antibiotics can result in lower competitive ability [34]. In addition to such trait-mediated effects, adaptation of a focal species to an abiotic stressor might influence biotic interactions by increasing its population size. Despite this range of possibilities of how adaptation to an abiotic stressor could affect biotic interactions, its ecological consequences have rarely been considered in a community context.

Here we used experimental evolution with freshwater algae to investigate if adaptation to abiotic change influences competitive interactions, and, vice versa, if competition influences adaptation to deteriorating environmental conditions. We used salt as a stressor because it has a negative effect on the fitness of freshwater algae, and because it can easily be manipulated without confounding changes in other abiotic factors. We did not intend to mimic a specific environmental change scenario observed in natural systems; rather we wanted to investigate conceptual questions about the interplay of competition and adaptation to an abiotic stressor. We conducted two sets of experiments (Fig. 1): in the first experiment we investigated if adaptation to salt in monoculture translated into increased population size (i.e. cell number) within salt-stressed mixtures of species, to test the hypothesis that competition can alter the effect of adaptation on population dynamics. In the second experiment we tested the hypotheses that interspecific competition constrains adaptation to salt stress, and that a history of salt stress leads to increased competitive ability at high salt. 


\section{Material and methods}

\section{Model organisms}

118 We tested our hypotheses using six species of microalgae from three different taxonomic

119 groups: the cyanobacteria Synechococcus leopoliensis (Canadian Phycological Culture Centre

120 (CPCC) 102) and Anabaena variabilis (CPCC 105), the diatoms Navicula pelliculosa (CPCC

121 552) and Nitzschia palea (CPCC 160), and the chlorophytes Pseudokirchneriella subcapitata

122 (CPCC 37) and Scenedesmus acutus (CPCC 10). We chose these six species because they

123 were available as axenic (i.e. bacteria-free) cultures and because we knew from previous

124 experiments that they grow well under the same experimental conditions $[35,36]$. The algae

125 were cultivated in Bold's basal medium [37], which was modified by adding silicate (0.58 g

$126 \mathrm{~L}^{-1}$ of $\mathrm{Na}_{2} \mathrm{SiO}_{3}$ ) and vitamins [38] to allow growth of diatoms. In all experiments, the algae

127 were cultivated at $25^{\circ} \mathrm{C}$ with light continuously provided at $100 \mu \mathrm{E} \mathrm{m}^{-2} \mathrm{~s}^{-1}$. Cultures were

128 axenic at the start of the experiment and propagated under sterile conditions. At the end of the

129 experiment, we plated a subset of the cultures (18 cultures of Anabaena and Scenedesmus) on

130 agar and observed no bacterial contaminations. We did not make cultures isogenic at the start

131 of the experiment; the observed evolutionary responses could thus have resulted either from

132 de novo mutations or from sorting of standing genetic variation.

In a preliminary experiment, we measured salt tolerance of the six species (Fig. S1),

134 and found a low limit of salt tolerance in Nitzschia and Pseudokirchneriella $\left(\sim 6 \mathrm{~g} \mathrm{NaCl} \mathrm{L}^{-1}\right)$,

135 intermediate salt tolerance in Anabaena, Scenedesmus and Navicula (limit: $\sim 14 \mathrm{~g} \mathrm{NaCl} \mathrm{L}^{-1}$ ),

136 and high salt tolerance in Synechococcus (limit: > $20 \mathrm{~g} \mathrm{NaCl} \mathrm{L}^{-1}$ ).

Adaptation to salt in monoculture

139 In a serial transfer experiment, we propagated monocultures of the six species with and

140 without increasing concentrations of $\mathrm{NaCl}$ (Fig. 1, Fig. S2). The algae were cultured in $125-$ 
$141 \mathrm{ml}$ glass flasks filled with $50 \mathrm{ml}$ of Bold's medium and continuously shaken at $250 \mathrm{rpm}$.

142 Every 3.5 days, we transferred $1 \mathrm{ml}$ of culture to $50 \mathrm{ml}$ of fresh medium for a total of 37

143 transfers ( 185 generations; one transfer corresponding to five generations). For each of the

144 six species, three replicate control lines were cultivated without addition of salt and three

145 replicate selection lines were cultivated with increasing salt. We increased the salt

146 concentration in the selection lines by $0.25 \mathrm{~g} \mathrm{~L}^{-1}$ every transfer until reaching $4 \mathrm{~g} \mathrm{NaCl} \mathrm{L}^{-1}$ at

147 transfer 16 (Fig. S2). We maintained this salt concentration for 10 transfers and then

148 continued the increase in salt until reaching the final concentration of $6 \mathrm{~g} \mathrm{NaCl} \mathrm{L}^{-1}$ at transfer

14934 , which we maintained for three more transfers. This salt concentration had a distinctly

150 negative effect on the growth rates of species with lowest salt tolerance but still allowed

151 persistence of all selection lines. A further increase in salt concentration would have probably

152 resulted in extinction of species with low tolerance and would thus have reduced the number

153 of species in the mixture assay.

154 The goal of the salt treatment was to simulate an environmental change scenario as it

155 would be experienced by the members of a community; we thus exposed all species to the

156 same change in environmental conditions rather than exposing each species to its respective

157 limit of salt tolerance. Because the species varied in salt tolerance (Fig. S1), they differed in

158 how strongly they were stressed by the salt treatment. Similarly, because the species varied in

159 growth rates and population sizes, they differed in the potential to adapt to salt. Such species-

160 specific differences are a property of any community and we thus made no attempt to reduce

161 these differences (e.g. by transferring equal cell numbers rather than equal volume).

$162 \quad$ After 37 transfers ( 185 generations), we measured population growth rate of each

163 line in the two final salt environments $\left(0\right.$ and $\left.6 \mathrm{~g} \mathrm{NaCl} \mathrm{L}^{-1}\right)$ to test if the species had adapted to

164 salt stress. Before starting the growth assay, we cultivated all lines at identical environmental

165 conditions for three transfer cycles, that is two cycles in the ancestral environment $(0 \mathrm{~g} \mathrm{NaCl}$ 
$\left.\mathrm{L}^{-1}\right)$ and one cycle in the assay environment $\left(0\right.$ and $\left.6 \mathrm{~g} \mathrm{NaCl} \mathrm{L}^{-1}\right)$, to reduce non-evolutionary

167 effects and pre-condition the lines to the assay conditions. We then inoculated flasks containing $50 \mathrm{ml}$ medium of either 0 or $6 \mathrm{~g} \mathrm{NaCl} \mathrm{L}^{-1}$ with $1 \mathrm{ml}$ of acclimated culture and measured absorbance through time. Depending on the time of individual cultures to reach carrying capacity, we measured absorbance over 2.5 to 7.5 weeks with 23 to 34 measurements. At each measurement, we removed $200 \mu 1$ of culture from each flask into 96well plates and measured absorbance at $660 \mathrm{~nm}$ on an optical plate reader (Synergy-HT,

173 BioTek, Winooski, VT). As done in other studies [8, 35], we calculated population growth

174 rates (see below) from raw absorbance values, i.e., we did not use standard curves to

175 transform absorbance to cell density. We thus did not take into account that changes in cell

176 size in response to salt might have influenced absorbance.

177

\section{Mixture assay}

To test if a history of salt stress can influence population size of species within salt-stressed mixtures of species, we assembled mixtures either of control or of selection lines, transplanted the mixtures into the two final salt environments of the selection experiment, and quantified species abundances over four transfers. Prior to the mixture assay, the lines had been cultivated for 37 transfers with or without increasing salt, followed by two transfers in the ancestral environment (see above). We assembled mixtures with equal biovolume of the six species. To this end, we determined species abundances in the monocultures by counting Lugol-fixed samples with an inverted microscope, and then converted abundances to biovolume using cellular biovolume estimates based on measurements of 20 individuals per species (Table S1) [39]. We assembled three replicate mixtures of control lines and three replicate mixtures of selection lines. From each of the six mixtures, we inoculated two flasks, one with a salt concentration of 0 and one of $6 \mathrm{~g} \mathrm{NaCl} \mathrm{L}^{-1}$. Every four days, we transferred 1 
$191 \mathrm{ml}$ of the mixtures into $50 \mathrm{ml}$ of fresh medium, with the flasks continuously shaken at 250

$192 \mathrm{rpm}$. Over the course of four transfers, we sampled the mixtures at each transfer, fixed the

193 samples with Lugol's solution, and counted species abundances with an inverted microscope.

194 We used different approaches to quantify population growth rate in the monoculture

195 assay and population size in the mixture assay (absorbance and abundance counts,

196 respectively) because high-throughput measurement of absorbance with a plate reader

197 allowed us to collect a dense time-series of absorbance data, while microscopical counts were

198 necessary to differentiate species in the mixture assay.

199

\section{Adaptation in monoculture vs. adaptation in pairs}

201 To test if competition can influence adaptation to salt stress, we propagated monocultures and 202 pairs of Anabaena and Scenedesmus with and without increasing salt for 46 transfers ( 230 generations), then re-isolated the two species and quantified their competitive ability with and without salt (Fig. 1). We chose the combination of Anabaena and Scenedesmus because in other species combinations the inferior competitor was outcompeted quickly both with and without increasing salt. In our experimental setup (i.e. a serial transfer experiment with

207 nutrient-replete conditions), competition is mediated by the difference in growth rates, with 208 the species with faster growth rate expected to outcompete the species with slower growth

209 rate. The monocultures and pairs of the two species were part of the selection experiment

210 described above and experienced the same changes in salt concentration except that cultures

211 spent nine more transfers at the final salt concentration (Fig. S2). To quantify how strongly

212 competition affected the abundances of the two species during the selection experiment, we

213 sampled the pairs and monocultures of Anabaena and Scenedesmus ten times over the course

214 of the selection experiment and microscopically determined the abundances of the two species 215 in Lugol-fixed samples. 
After 46 transfers in the selection experiment, we re-isolated Anabaena and

Scenedesmus by plating the cultures on agar and isolating individual colonies. Monocultures in the assay environment) to reduce non-evolutionary effects.

The pre-conditioned cultures were used to start competition assays to test if competition history and salt history influenced competitive ability. To this end, we assembled pairs of

Anabaena and Scenedesmus in the two final salt environments and determined changes in

biomass of the two species through time. We conducted two competition assays that differed in how we assembled the pairs (Fig. 1). First, we assembled pairs of co-evolved Anabaena and Scenedesmus, i.e. lines that had the same competition and salt history. However, because

232 pairs differed in both competitors in this assay (Fig. 1), differences among treatments could not unequivocally be attributed to one of the two species. We thus conducted a second reference line, we randomly selected one of the three replicate control lines (i.e. competition

237 history: monoculture, salt history: no salt). The second competition assay was started three 238 weeks after the first competition assay; cultures were stored meanwhile as monocultures at 239 their respective salt selection environment and then acclimated for one cycle to the salt assay 240 environment. 
In the competition assays, we measured chlorophyll a concentrations of the two

competitors as proxy for biomass using a FluoroProbe (bbe Moldaenke, Kiel-Kronshagen,

243 Germany), a fluorometer which can discriminate among algal groups [40]. Before the start of

244 the assay, we calibrated the FluoroProbe with cultures of Anabaena and Scenedesmus with

245 known chlorophyll a concentrations. We started the competition assay with equal chlorophyll

246 a concentrations $(2 \mu \mathrm{g})$ of both species in flasks filled with $50 \mathrm{~mL}$ of Bold's medium. We

247 transferred the cultures every 3.5 days for ten transfers in the first competition assay and for

248 four transfers in the second competition assay and measured chlorophyll a concentrations of

249 both species at each transfer.

Data analysis

All analyses were computed with $\mathrm{R}$ version 3.6.2 [41]. We estimated population

253 growth rates (absorbance day ${ }^{-1}$ ) in monoculture assays by fitting logistic growth curves to

254 absorbance vs. time curves using the R package grofit [42]. Growth curves could not be fit

255 when absorbance did not increase; in this case (the three control lines of Nitzschia at $6 \mathrm{~g} \mathrm{NaCl}$

$256 \mathrm{~L}^{-1}$ ) growth rate was set to zero. Some of the growth curves of Anabaena were difficult to fit

257 because this species sometimes grew in clumps at high salt, which led to unreliable

258 absorbance measurements. We thus do not present monoculture growth rates of Anabaena.

259 To investigate treatment effects on growth rates, we computed two-way ANOVAs for

260 each of the five species using assay environment and salt history as explanatory variables. It

261 would have been preferential to include 'line' as a random effect because each of the six lines

262 (three control lines, three selection lines) was assayed in two environments (with and without

263 salt, respectively). However, computation of linear mixed models with 'line' as a random

264 effect often resulted in singular fits because of zero or almost zero variation among lines. We

265 thus dropped the random effect and computed ANOVAs instead. In case of a significant 
interaction, we additionally computed two separate ANOVAs for each assay environment

with salt history as the explanatory variable. We used the function $l m$ to compute ANOVAs and the function Anova in the car package to calculate P-values. mixture assay as response variable, and assay environment and salt history as explanatory variables. Abundances were ln-transformed prior to analyses, except for abundances of Pseudokirchneriella which remained untransformed due to better fit with model assumptions. competition history, salt history and assay environment on relative chlorophyll a (arcsine square-root transformed) in the competition assay. In all our analyses, we did not use model selection to identify important predictor variables, but included all factors manipulated in the experimental design.

\section{Results}

\section{Adaptation of monocultures}

284 After 185 generations of growth with and without increasing salt, respectively, we detected 285 adaptation to salt in two of five species, Nitzschia and Pseudokirchneriella (Fig. 2, Table S2), 286 that is, lines with a history of salt stress had higher population growth rates at $6 \mathrm{~g} \mathrm{NaCl} \mathrm{L}^{-1}$ 287 than lines without a history of salt stress (ANOVA: Nitzschia: $\mathrm{F}_{1,4}=16.19, \mathrm{P}=0.016$; 288 Pseudokirchneriella: $\left.\mathrm{F}_{1,4}=77.70, \mathrm{P}=0.001\right)$. In the no-salt assay environment, however, salt

289 history had no effect on the growth rates of these two species (ANOVA: Nitzschia: $\mathrm{F}_{1,4}=0.64$, $290 \mathrm{P}=0.467 ;$ Pseudokirchneriella: $\mathrm{F}_{1,4}=2.78, \mathrm{P}=0.171$ ). Among the remaining three species, 
291 Scenedesmus and Navicula had reduced growth rates in the high-salt assay environment environment (Fig. 2, Table S2).

294

\section{Mixture assay}

When assaying mixtures of either control or selection lines, we found that the effect of salt history varied among species and depended on assay environment (Fig. 3 a-c, Table S3). Most species declined in abundance over the four transfers of the assay, except for Synechococcus, which increased (Fig. S3). Salt history affected the abundance of three species: Nitzschia, Pseudokirchneriella, Scenedesmus (Fig. 3 a-c, Table S3). In Nitzschia, lines with a history of salt stress had lower average abundance than control lines when assayed without salt (58\% lower abundance; $\left.\mathrm{F}_{1,4}=25.97, \mathrm{P}=0.007\right)$. At high salt, however, the effect of salt history on average abundance of Nitzschia was not significant $\left(\mathrm{F}_{1,4}=4.54, \mathrm{P}=0.10\right)$ and control and selection lines declined similarly fast (Fig. S3a). In Pseudokirchneriella, selection lines had lower average abundance than control lines in the no-salt assay environment (24\% lower abundance; $\left.\mathrm{F}_{1,4}=24.11, \mathrm{P}=0.008\right)$, but 2.5 times higher abundance than control lines in the high-salt assay environment $\left(\mathrm{F}_{1,4}=14.90, \mathrm{P}=0.018\right)$. However, these treatment effects on Pseudokirchneriella did not persist until the end of the mixture assay: within four transfers, Pseudokirchneriella went extinct or declined to low abundance irrespective of salt history and assay environment (Fig. S3b). In Scenedesmus, a history of salt stress had no effect on average abundance in the no-salt assay environment $\left(\mathrm{F}_{1,4}=1.73, \mathrm{P}=0.258\right)$, but a positive effect at high salt (1.7 times higher abundance; $\left.\mathrm{F}_{1,4}=11.86, \mathrm{P}=0.026\right)$. Among the remaining three species, abundance of Anabaena was negatively affected by salt irrespective of salt history (Fig. S3c, Table S3), while Navicula and Synechococcus were unaffected by both salt history and assay environment (Fig. 3d, e, Table S3). 
Adaptation in monoculture vs. adaptation in pairs

Interspecific competition strongly influenced species abundances during the selection

experiment, but did not lead to any evolutionary responses. In the selection experiment,

Anabaena reduced the abundance of Scenedesmus as long as salt concentration was low (Fig.

S4b). However, at the final salt concentration of $6 \mathrm{~g} \mathrm{NaCl} \mathrm{L}^{-1}$ the competitive hierarchy

reversed and Anabaena was negatively affected by Scenedesmus (Fig. S4a). This change in

Anabaena dominated without salt (Fig. 4a), while Scenedesmus dominated at high salt (Fig.

4b), irrespective of competition history.

The two competition assays revealed that lines of Anabaena with a history of salt stress had reduced competitive ability in the no-salt assay environment. When assayed with co-evolved competitors, high-salt lines of Anabaena had lower relative chlorophyll a than no-

0.002; Table S4, Fig. S5). Because both competitors differed among treatment combinations, assay: $\mathrm{F}_{1,16}=5.78, \mathrm{P}=0.029$, Table S5, Fig. 4a). As a consequence, in the no-salt assay environment the biomass (i.e. chlorophyll a concentration) of the competing reference line

\section{Discussion}


341 With our experiments we addressed a range of scenarios that can arise from the interplay of

342 competition and adaptation to an abiotic stressor. In the first experiment, we found that

343 competition can alter how adaptation to an abiotic stressor influences population dynamics

344 (Fig. 2, 3): effects of salt history on population growth rate in monoculture did not necessarily

345 lead to increased population size within communities. Conversely, some evolutionary effects,

346 specifically, reduced population size of selection lines without salt, appeared only within

347 mixtures of species, not in monoculture. In the second experiment, we found no effect of

348 competition on adaptation to salt stress (Fig. 4). However, salt history influenced competition:

349 after selection at increasing salt, one of two species had reduced competitive ability without

350 salt (Fig. 4a). Collectively, our results highlight that ecological and evolutionary processes

351 interactively shape communities in changing environments, but that these eco-evolutionary

352 effects can be diverse and species-specific.

354 lowest limit of salt tolerance adapted to salt (Fig. 2a, b), while species that were less

355 negatively affected by salt showed no sign of adaptation (Fig. 2 c-e). This finding is in line

356 with the prediction that species with narrow tolerance of a stressor should evolve more than

357 species with broader tolerance because they experience stronger selection pressure [43].

358 Species-specific differences in genetic variance and covariance could be additional reasons

359 for variation among species in the rate of evolution [43, 44]. Accordingly, an experiment that

360 investigated thermal evolution in three phytoplankton species found largest fitness

361 improvements in the species with highest population density and the least complex genome

362 [45]. Given that only two species adapted in our experiment, our sample size is not large

363 enough to evaluate if species-specific differences other than ecological tolerance influenced

364 the evolutionary response to salt. Such variation among species in the ability to adapt to

365 environmental change could add further complexity to adaptation in a community context. 
Our experiments suggest that competition can modulate how adaptation to abiotic change influences population dynamics. When assayed at high salt in monoculture, two species (Nitzschia and Pseudokirchneriella) showed signatures of adaptation, that is, higher population growth rate of selection lines than control lines (Fig. 2a, b). When assayed at high salt within mixtures, however, only one of these two species (Pseudokirchneriella) had higher population size of selection than control lines (Fig. 3b), and this positive effect of salt history was only transient (Fig. S3b). In contrast, control and selection lines of Nitzschia had similar population size and declined equally fast within salt-stressed mixtures (Fig. 3a, Fig. S3a). population size in acidified communities [20]. Such a pattern can emerge when competition changes the fitness landscape [18]; thereby adaptation of a species to abiotic change improves

377 its fitness only in the same community context in which it has evolved [18]. In our mixtures, low competitive ability of the two species that adapted to salt might have also contributed to this pattern. Nitzschia and Pseudokirchneriella were the weakest competitors in the community and declined to extinction or to low abundance irrespective of salt history and salt

381 environment (Fig. S3). It remains to be determined if effects of adaptation on population 382 dynamics are less contingent on community context in stronger competitors. In addition to 383 low competitive ability, low starting abundance could have contributed to the fast extinction 384 of Nitzschia: this species was the largest in our community (Table S1) and was thus 385 inoculated with the lowest abundance among the six species. Taken together, the positive 386 effect of adaptation to abiotic change on population size may be reduced when community 387 context changes; such a scenario could result from immigration of novel competitors in response to environmental change $[27,28,46]$. 
in the no-salt environment when these species were assayed within mixtures (Fig. 3a, b), but not when assayed in isolation (Fig. 2a, b). A possible mechanism underlying such a pattern is a trade-off between traits that mediate stress tolerance and competition [34]. We did not measure traits and can thus only speculate about potential mechanisms. Tolerance of high salt concentrations is conferred by the ability to efficiently export ions $\left(\mathrm{Na}^{+}\right.$and $\left.\mathrm{Cl}^{-}\right)$and accumulate compatible solutes, i.e. organic molecules that maintain osmotic balance without interfering with metabolism [47]. High energetic costs for ion export and for synthesis of compatible solutes [48] may result in reduced competitive ability. However, when adaptation to an abiotic stressor occurs within communities, rather than in isolation as in our experiment, reduced competitive ability might not evolve. Our experiment thus simulates a scenario in which environmental change is followed by a change in community context, which could result from immigration of novel competitors or from changes in local community composition due to species-specific differences in environmental tolerance. To elucidate if adaptation to an abiotic stressor is associated with changes in traits that mediate biotic interactions, a trait-based approach would be an interesting avenue for future experiments. Competition had no effect on adaptation to salt stress (Fig. 4). We had predicted that competition during the selection experiment would constrain adaptation to salt because of reduced population size and/or increased genetic constraints [23, 26]. Alternatively, competition could facilitate adaptation to abiotic stress by increasing the strength of selection [25]. The two species that we used to test this question, Anabaena and Scenedesmus, adapted to salt neither in monoculture nor in pairs. Similarly, an experiment that manipulated $\mathrm{CO}_{2}$ enrichment and competition in a selection experiment with two algae species found neither adaptation to $\mathrm{CO}_{2}$ nor to competition [19]. In our experiment, low selection pressure could explain why the monocultures did not adapt to salt, given that the two species had not reached their limit of salt tolerance in the selection experiment. However, the combination of salt and 
competition in the selection experiment drove the inferior competitor to the brink of

extinction (Fig. S4), suggesting that the inferior competitor experienced strong selection

Maybe relaxed selection pressure on Scenedesmus towards the end of the experiment and only evolutionary response of these two species. competitive ability at high salt. In contrast, however, strains of Anabaena with a history of salt stress had reduced competitive ability in the no-salt assay environment. Such a pattern is an indication of conditionally deleterious mutations [49], i.e. mutations that are neutral in the molecular nor trait data, we cannot elucidate potential mechanisms behind the observed maladaptation in the ancestral environment. The evolutionary response of Anabaena was independent of competition history and of whether fitness was assayed in competition with a response to salt is also illustrated by the response of the competing reference line: in the no436 salt assay, the chlorophyll a concentration attributable to the reference line of Scenedesmus 437 was 1.9 times higher when competing with selection lines of Anabaena than when competing 438 with control lines (Fig. S6a). Overall, our results suggest that evolutionary responses to 439 abiotic change can influence biotic interactions, such that populations with different histories 440 of abiotic stress might vary in their effects on communities and ecosystems. 
The emerging field of eco-evolutionary dynamics investigates the effects of evolution on populations, communities, and ecosystems [6]. Research on adaptation to abiotic change usually explores the effect of evolution on population dynamics $[4,7,11]$, while studies that investigate the effect of evolution on species interactions often focus on biotic selective agents [50-52]. Here we showed that evolutionary responses to an abiotic selective agent can alter biotic interactions (Fig. 4a, Fig. S6a). Moreover, we found that competition can modulate the effect of adaptation on population dynamics (Fig. 2, 3), suggesting that adaptation to abiotic change may not enhance performance of a species when community context changes [18] e.g. because of immigrating competitors [28]. We have observed a range of complexities arising from adaptation in a multi-species context despite using a fairly simple model system. Higher

diversity and trophic structure of natural systems will pose an even greater challenge to understanding eco-evolutionary dynamics in changing environments [33, 53]. Environmental

453 fluctuation is another complexity of natural systems that we were not able to address in our experiment. Temporary environmental amelioration could promote adaptation to abiotic change by increasing population size and thus the supply of beneficial mutations [11], but could also reduce the potential for adaptation by relaxing selection pressure [54]. Taking these complexities into account will be crucial in future work to further increase our understanding of adaptation to abiotic change in a community context.

\section{Acknowledgements}

We thank Etienne Low-Décarie and Graham Bell for input on the design and analysis of

464 the experiment, and Yinci Yan for help with the implementation of the experiment. 
466

467

468

469

470

471

472

473

474

475

476

477

478

479

480

481

482

483

484

485

486

487

488

489

490

\section{Funding}

This work was supported by an Erwin-Schrödinger scholarship of the Austrian Science Fund (FWF), grant no. J3265-B16 to R.L., and an NSERC Discovery Grant to G.F.F.

\section{References}

1. Ceballos G., Ehrlich P.R. 2002 Mammal population losses and the extinction crisis. Science 296, 904-907. (doi:10.1126/science.1069349).

2. Both C., Bouwhuis S., Lessells C.M., Visser M.E. 2006 Climate change and population declines in a long-distance migratory bird. Nature 441, 81-83. (doi:10.1038/nature04539).

3. Stuart S.N., Chanson J.S., Cox N.A., Young B.E., Rodrigues A.S.L., Fischman D.L., Waller R.W. 2004 Status and trends of amphibian declines and extinctions worldwide. Science 306, 1783-1786. (doi:10.1126/science.1103538).

4. Bell G., Gonzalez A. 2009 Evolutionary rescue can prevent extinction following environmental change. Ecol Lett 12, 942-948. (doi:10.1111/j.1461-0248.2009.01350.x).

5. Gomulkiewicz R., Holt R.D. 1995 When does evolution by natural selection prevent extinction? Evolution 49, 201-207. (doi:10.2307/2410305).

6. Hendry A.P. 2016 Eco-evolutionary dynamics. Princeton and Oxford, Princeton University Press; 416 p.

7. Lohbeck K.T., Riebesell U., Reusch T.B.H. 2012 Adaptive evolution of a key phytoplankton species to ocean acidification. Nat Geosci 5, 346-351. (doi:10.1038/ngeo1441). 8. Lachapelle J., Bell G. 2012 Evolutionary rescue of sexual and asexual populations in a deteriorating environment. Evolution 66, 3508-3518. (doi:10.1111/j.15585646.2012.01697.x) 
9. $\quad$ Reid N.M., Proestou D.A., Clark B.W., Warren W.C., Colbourne J.K., Shaw J.R.,

492 Karchner S.I., Hahn M.E., Nacci D., Oleksiak M.F., et al. 2016 The genomic landscape of

493 rapid repeated evolutionary adaptation to toxic pollution in wild fish. Science 354, 1305-1308.

494 (doi:10.1126/science.aah4993).

495 10. Collins S., de Meaux J. 2009 Adaptation to different rates of environmental change in

496 Chlamydomonas. Evolution 63, 2952-2965. (doi:10.1111/j.1558-5646.2009.00770.x).

497 11. Schaum C.E., Buckling A., Smirnoff N., Studholme D.J., Yvon-Durocher G. 2018

498 Environmental fluctuations accelerate molecular evolution of thermal tolerance in a marine

499 diatom. Nat Commun 9, 1719. (doi:10.1038/s41467-018-03906-5).

500 12. Schaum C.E., Barton S., Bestion E., Buckling A., Garcia-Carreras B., Lopez P., Lowe

501 C., Pawar S., Smirnoff N., Trimmer M., et al. 2017 Adaptation of phytoplankton to a decade

502 of experimental warming linked to increased photosynthesis. Nat Eco Evo 1, 0094.

503 (doi:10.1038/s41559-017-0094).

504 13. Lawrence D., Fiegna F., Behrends V., Bundy J.G., Phillimore A.B., Bell T.,

505 Barraclough T.G. 2012 Species interactions alter evolutionary responses to a novel

506 environment. PLoS Biology 10, e1001330. (doi:10.1371/journal.pbio.1001330).

507 14. Zuppinger-Dingley D., Schmid B., Petermann J.S., Yadav V., De Deyn G.B., Flynn

508 D.F.B. 2014 Selection for niche differentiation in plant communities increases biodiversity

509 effects. Nature 515, 108-111. (doi:10.1038/nature13869).

510 15. Castledine M., Padfield D., Buckling A. 2020 Experimental (co)evolution in a multi-

511 species microbial community results in local maladaptation. Ecol Lett 23, 1673-1681.

512 (doi:10.1111/ele.13599).

513 16. Hiltunen T., Cairns J., Frickel J., Jalasvuori M., Laakso J., Kaitala V., Künzel S.,

514 Karakoc E., Becks L. 2018 Dual-stressor selection alters eco-evolutionary dynamics in

515 experimental communities. Nat Eco Evo 2, 1974-1981. (doi:10.1038/s41559-018-0701-5). 
516 17. Collins S. 2011 Competition limits adaptation and productivity in a photosynthetic

517 alga at elevated $\mathrm{CO}_{2}$. Proc $R$ Soc B 278, 247-255. (doi:10.1098/rspb.2010.1173).

518 18. Kleynhans E.J., Otto S.P., Reich P.B., Vellend M. 2016 Adaptation to elevated $\mathrm{CO}_{2}$ in

519 different biodiversity contexts. Nat Commun 7, 12358. (doi:10.1038/ncomms12358).

520 19. Listmann L., Hattich G.S.I., Matthiessen B., Reusch T.B.H. 2020 Eco-evolutionary

521 interaction in competing phytoplankton: nutrient driven genotype sorting likely explains

522 dominance shift and species responses to $\mathrm{CO}_{2}$. Front Mar Sci 7.

523 (doi:10.3389/fmars.2020.00634).

524 20. Bach L.T., Lohbeck K.T., Reusch T.B.H., Riebesell U. 2018 Rapid evolution of highly

525 variable competitive abilities in a key phytoplankton species. Nat Eco Evo 2, 611-613.

526 (doi:10.1038/s41559-018-0474-x).

527 21. Tatters A.O., Schnetzer A., Fu F., Lie A.Y.A., Caron D.A., Hutchins D.A. 2013 Short-

528 versus long-term responses to changing $\mathrm{CO}_{2}$ in a coastal dinoflagellate bloom: implications

529 for interspecific competitive interactions and community structure. Evolution 67, 1879-1891.

530 (doi:10.1111/evo.12029).

$531 \quad 22 . \quad$ Bocedi G., Atkins K.E., Liao J.S., Henry R.C., Travis J.M.J., Hellmann J.J. 2013

532 Effects of local adaptation and interspecific competition on species' responses to climate 533 change. Ann N Y Acad Sci 1297, 83-97. (doi:10.1111/nyas.12211).

534 23. Johansson J. 2008 Evolutionary responses to environmental changes: How does

535 competition affect adaptation? Evolution 62, 421-435. (doi:10.1111/j.1558-

536 5646.2007.00301.x).

537 24. de Mazancourt C., Johnson E., Barraclough T.G. 2008 Biodiversity inhibits species'

538 evolutionary responses to changing environments. Ecol Lett 11, 380-388.

539 (doi:10.1111/j.1461-0248.2008.01152.x). 
25. Osmond M.M., de Mazancourt C. 2013 How competition affects evolutionary rescue.

Phil Trans R Soc B 368, 20120085. (doi:10.1098/rstb.2012.0085).

26. Lau J.A., Terhorst C.P. 2015 Causes and consequences of failed adaptation to

543 biological invasions: the role of ecological constraints. Mol Ecol 24, 1987-1998.

544 (doi:10.1111/mec.13084).

545 27. Urban M.C., De Meester L., Vellend M., Stoks R., Vanoverbeke J. 2012 A crucial step

546 toward realism: responses to climate change from an evolving metacommunity perspective.

547 Evol Appl 5, 154-167. (doi:10.1111/j.1752-4571.2011.00208.x).

548 28. De Meester L., Vanoverbeke J., Kilsdonk L.J., Urban M.C. 2016 Evolving

549 perspectives on monopolization and priority effects. Trends Ecol Evol 31, 136-146.

$550 \quad$ (doi:10.1016/j.tree.2015.12.009).

551 29. Gomez P., Paterson S., De Meester L., Liu X., Lenzi L., Sharma M.D., McElroy K.,

552 Buckling A. 2016 Local adaptation of a bacterium is as important as its presence in

553 structuring a natural microbial community. Nat Commun 7, 12453.

554 (doi:10.1038/ncomms12453).

555 30. Pantel J.H., Duvivier C., De Meester L. 2015 Rapid local adaptation mediates

556 zooplankton community assembly in experimental mesocosms. Ecol Lett 18, 992-1000.

557 (doi:10.1111/ele.12480).

558 31. terHorst C.P., Lennon J.T., Lau J.A. 2014 The relative importance of rapid evolution

559 for plant-microbe interactions depends on ecological context. Proc R Soc B 281, 20141615.

560 (doi:10.1098/rspb.2014.0028).

561 32. Matthews B., Narwani A., Hausch S., Nonaka E., Peter H., Yamamichi M., Sullam

562 K.E., Bird K.C., Thomas M.K., Hanley T.C., et al. 2011 Toward an integration of

563 evolutionary biology and ecosystem science. Ecol Lett 14, 690-701. (doi:10.1111/j.1461-

$564 \quad$ 0248.2011.01627.x). 
33. Hendry A.P. 2019 A critique for eco-evolutionary dynamics. Funct Ecol 33, 84-94.

566

567

568

569

570

571

572

573

574

575

576

577

578

579

580

581

582

583

584

585

586

587

588

589 (doi:10.1111/1365-2435.13244).

34. Phan K., Ferenci T. 2013 A design-constraint trade-off underpins the diversity in ecologically important traits in species Escherichia coli. Isme J 7, 2034-2043.

(doi:10.1038/ismej.2013.82).

35. Low-Décarie E., Jewell M.D., Fussmann G.F., Bell G. 2013 Long-term culture at elevated atmospheric $\mathrm{CO} 2$ fails to evoke specific adaptation in seven freshwater phytoplankton species. Proc R Soc B 280. (doi:10.1098/rspb.2012.2598).

36. Limberger R., Low-Décarie E., Fussmann G.F. 2014 Final thermal conditions override the effects of temperature history and dispersal in experimental communities. Proc R Soc B 281, 20141540. (doi:10.1098/rspb.2014.1540).

37. Bold H. 1949 The morphology of Chlamydomonas chlamydogama, sp. nov. Bull Torrey Bot Club 76, 101-108. (doi:10.2307/2482218).

38. Low-Décarie E., Fussmann G.F., Bell G. 2011 The effect of elevated $\mathrm{CO}_{2}$ on growth and competition in experimental phytoplankton communities. Glob Change Biol 17, 25252535. (doi:10.1111/j.1365-2486.2011.02402.x ).

39. Hillebrand H., Durselen C.D., Kirschtel D., Pollingher U., Zohary T. 1999 Biovolume calculation for pelagic and benthic microalgae. J Phycol 35, 403-424. (doi:10.1046/j.15298817.1999.3520403.x).

40. Catherine A., Escoffier N., Belhocine A., Nasri A.B., Hamlaoui S., Yepremian C., Bernard C., Troussellier M. 2012 On the use of the FluoroProbe®, a phytoplankton quantification method based on fluorescence excitation spectra for large-scale surveys of lakes and reservoirs. Water Res 46, 1771-1784. (doi:10.1016/j.watres.2011.12.056).

41. R Core Team. 2015 R: A language and environment for statistical computing. R foundation for statistical computing. (Vienna, Austria. URL http://www.R-project.org/. 
42. Kahm M., Hasenbrink G., Lichtenberg-Frate H., Ludwig J., Kschischo M. 2010 grofit:

591 fitting biological growth curves with R. Journal of Statistical Software 33, 1-21.

592 43. Lande R. 1979 Quantitative genetic analysis of multivariate evolution, applied to

593 brain:body size allometry. Evolution 33, 402-416. (doi:10.2307/2407630).

594 44. Barraclough T.G. 2015 How do species interactions affect evolutionary dynamics 595 across whole communities? Annu Rev Ecol Evol Syst 46, 25-48. (doi:10.1146/annurev596 ecolsys-112414-054030).

597 45. Barton S., Jenkins J., Buckling A., Schaum C.E., Smirnoff N., Raven J.A., Yvon598 Durocher G. 2020 Evolutionary temperature compensation of carbon fixation in marine 599 phytoplankton. Ecol Lett 23, 722-733. (doi:10.1111/ele.13469).

600 46. Alexander J.M., Diez J.M., Levine J.M. 2015 Novel competitors shape species' 601 responses to climate change. Nature 525, 515-518. (doi:10.1038/nature14952).

602 47. Hagemann M. 2011 Molecular biology of cyanobacterial salt acclimation. Fems 603 Microbiology Reviews 35, 87-123. (doi:10.1111/j.1574-6976.2010.00234.x).

604 48. Oren A. 1999 Bioenergetic aspects of halophilism. Microbiol Mol Biol R 63, 334-+. 605 (doi:10.1128/mmbr.63.2.334-348.1999).

606 49. Mee J.A., Yeaman S. 2019 Unpacking conditional neutrality: genomic signatures of 607 selection on conditionally beneficial and conditionally deleterious mutations. Am Nat 194, 608 529-540. (doi:10.1086/702314).

609 50. Yoshida T., Jones L.E., Ellner S.P., Fussmann G.F., Hairston N.G.J. 2003 Rapid 610 evolution drives ecological dynamics in a predator-prey system. Nature 424, 303-306. 611 (doi:10.1038/nature01767).

612 51. Frickel J., Sieber M., Becks L. 2016 Eco-evolutionary dynamics in a coevolving host613 virus system. Ecol Lett 19, 450-459. (doi:10.1111/ele.12580). 
614 52. Hart S.P., Turcotte M.M., Levine J.M. 2019 Effects of rapid evolution on species

615 coexistence. 116, 2112-2117. (doi:10.1073/pnas.1816298116).

616 53. De Meester L., Brans K.I., Govaert L., Souffreau C., Mukherjee S., Vanvelk H.,

617 Korzeniowski K., Kilsdonk L., Decaestecker E., Stoks R., et al. 2019 Analysing eco-

618 evolutionary dynamics - the challenging complexity of the real world. Funct Ecol 33, 43-59.

619 (doi:10.1111/1365-2435.13261).

620 54. Hao Y.-Q., Brockhurst M.A., Petchey O.L., Zhang Q.-G. 2015 Evolutionary rescue

621 can be impeded by temporary environmental amelioration. Ecol Lett 18, 892-898.

622 (doi:10.1111/ele.12465).

623

624 


\section{Figure legends}

626 Figure 1: Experimental design. In Experiment 1, we propagated monocultures of six species

627 of microalgae with or without increasing salt for $\sim 185$ generations, and then quantified

628 growth rate in monoculture and population size in mixtures of species with and without salt.

629 White boxes are flasks without salt, grey boxes are flasks with salt. In Experiment 2, we

630 propagated two species in monoculture and in pairs, with and without salt. After $\sim 230$

631 generations we re-isolated the species on agar plates and quantified the chlorophyll-a

632 concentration (as proxy for biomass) of all lines when competing either with a competitor of

633 the same salt and competition history (co-evolved competitor) or with a reference line (naïve

634 competitor; salt history: no salt, competition history: monoculture). Bold species

635 abbreviations denote lines cultivated in pairs during the selection experiment.

636

637 Figure 2: Effects of salt history and assay environment on the monoculture growth rates of

638 five species of microalgae (sorted by salt tolerance). Control lines (blue) have a salt history of

63937 transfers at $0 \mathrm{~g} \mathrm{NaCl} \mathrm{L}^{-1}$, selection lines (red) have a salt history of 37 transfers at

640 increasing salt stress with a final concentration of $6 \mathrm{~g} \mathrm{NaCl} \mathrm{L}^{-1}$. Salt concentrations in the two

641 assay environments were the same as in the final selection environments, i.e. 0 and $6 \mathrm{~g} \mathrm{NaCl}$

$642 \mathrm{~L}^{-1}$, respectively. Inserts give the P-values for assay environment $(\mathrm{A})$, salt history $(\mathrm{H})$, and

643 their interaction $(\mathrm{AxH})$. Bold font denotes P-values < 0.05. For F-statistics see Table S2.

$644 \quad$ Values are means $\pm \mathrm{SE}, \mathrm{n}=3$

646 Figure 3: Effects of salt history and assay environment on species abundances in the mixture

647 assay. Mixtures of six species were assembled either from control lines (blue) or from

648 selection lines (red). Species abundances were averaged over four transfers in assay

649 environments with and without salt, respectively. Inserts give the P-values for assay 
650 environment (A), salt history $(\mathrm{H})$, and their interaction $(\mathrm{AxH})$. For F-statistics see Table S3.

651 Abundances were ln-transformed prior to analyses except for Pseudokirchneriella. For

652 consistency with Fig. 2, we do not show Anabaena, which was also part of the communities.

653 For time-resolved data of all six species, see supplementary figure S4. Values are means \pm SE

654 (SE too small to be visible), $\mathrm{n}=3$.

655

656 Figure 4: Effects of competition history, salt history, and assay environment on competitive

657 abilities of Anabaena and Scenedesmus. Relative chlorophyll a of (a) Anabaena in

658 competition with a reference line of Scenedesmus, and (b) of Scenedesmus in competition

659 with a reference line of Anabaena. Values are means $\pm \mathrm{SE}, \mathrm{n}=3$.

660

661 
Experiment 1: Does competition alter the effect of adaptation on population dynamics?

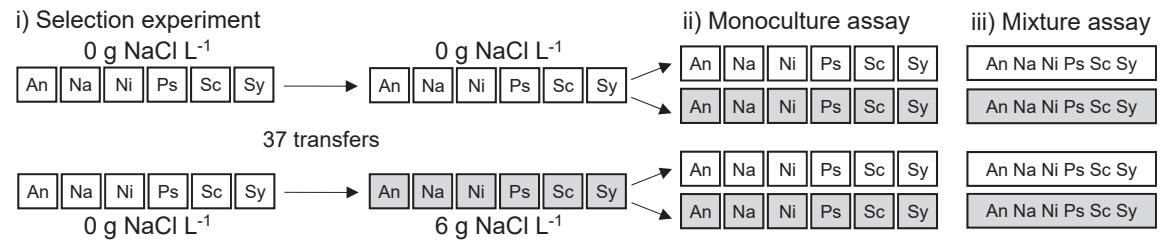

Experiment 2: Does competition affect adaptation to salt? Does salt history affect competition? i) Selection experiment $\quad$ ii) Re-isolation iii) Competition assay iv) Competition assay

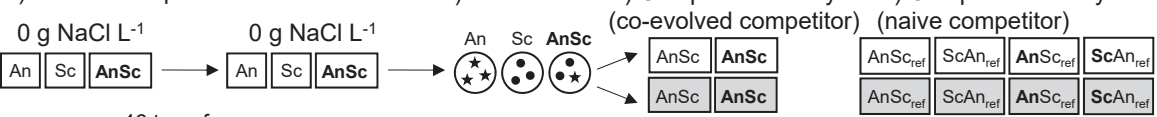

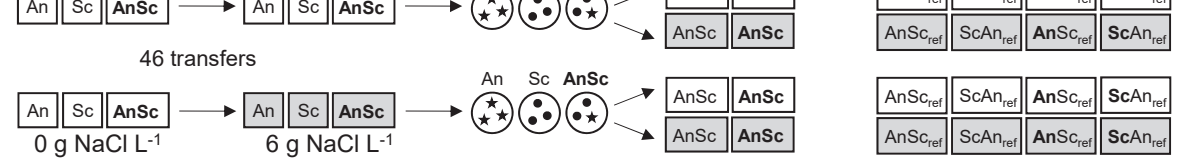




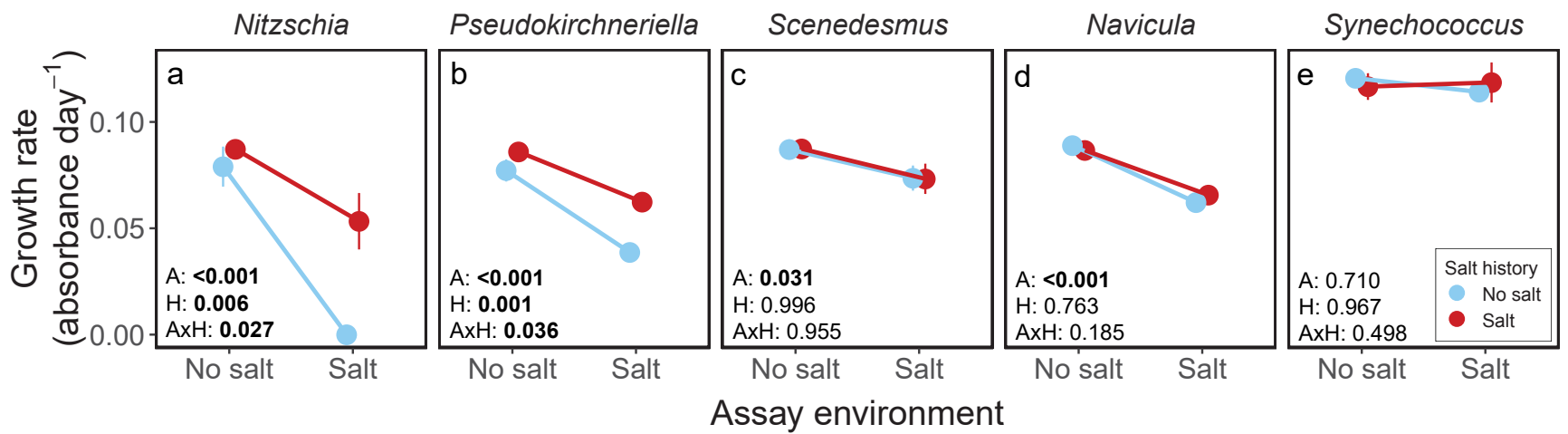




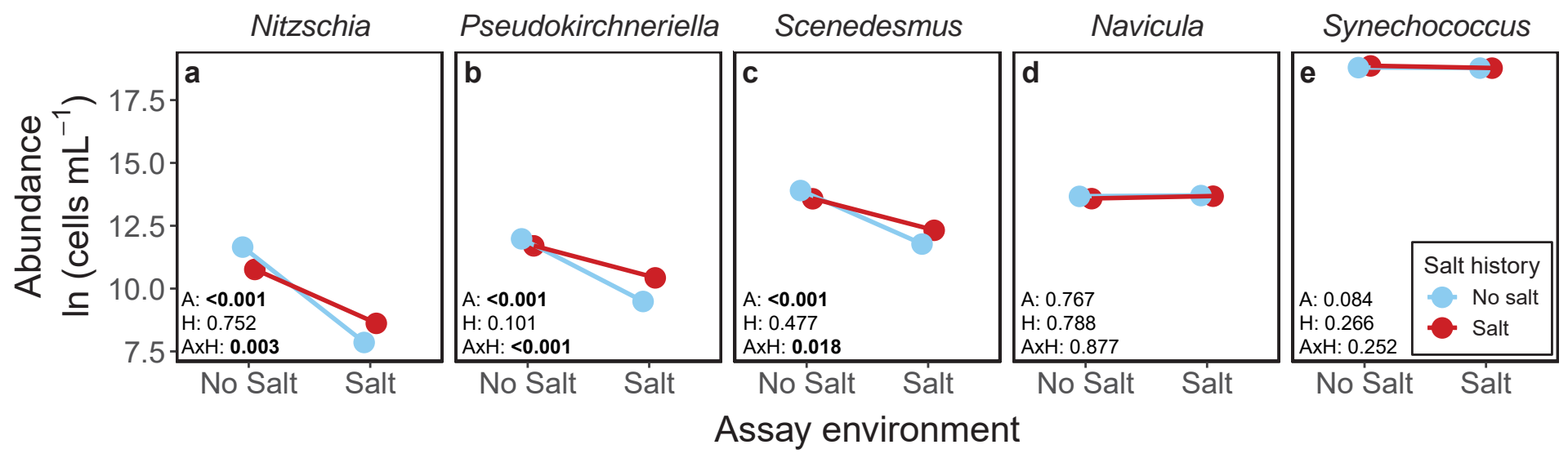




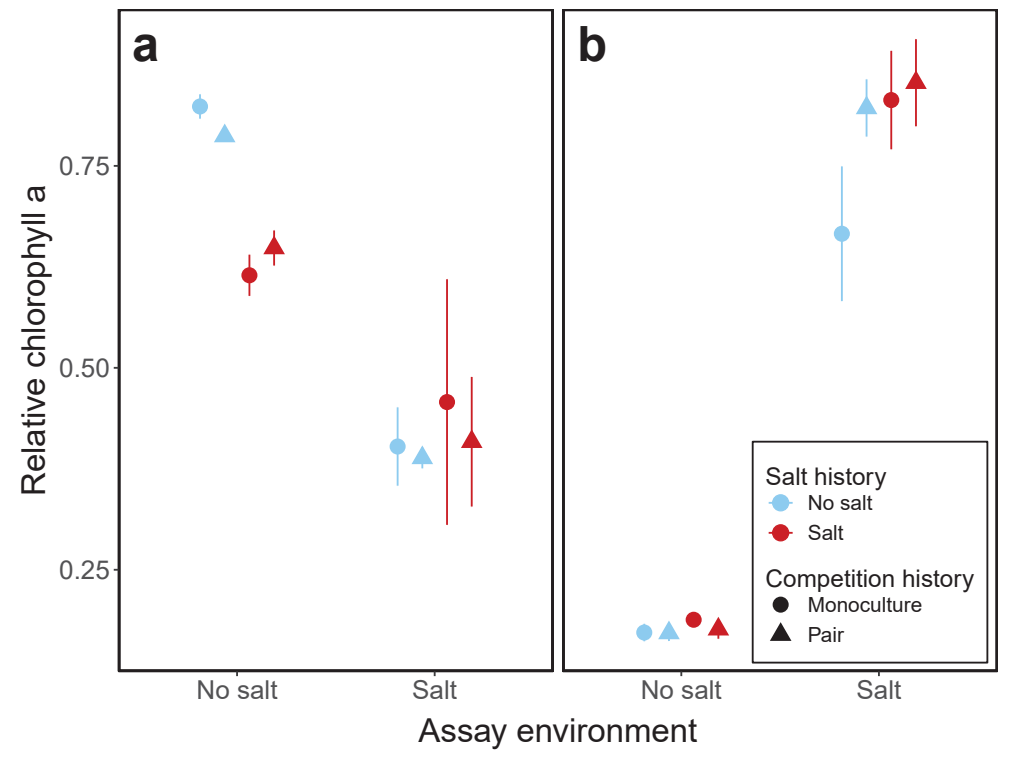

